\title{
Kikuchi's disease: A case report from south India
}

\author{
Sudhakar MK, Sathyamurthy P, Indhumathi E \\ Amarabalan Rajendran, Bavya Vivek
}

\begin{abstract}
Introduction: Kikuchi-Fujimoto disease (KFD), or histiocytic necrotizing lymphadenitis, is a rare benign, self-limiting cervical lymphadenitis of unknown etiology. It predominantly affects young women and can closely mimic infective and immunological disorders. Case Report: We report a 25 yr old female who presented with fever, polyarthritis and cervical lymphadenopathy. She had multiple enlarged cervical nodes. Examination of other systems was normal. Laboratory investigations were also normal. Fine needle aspiration cytology of the cervical node showed features suggestive of reactive lymphadenitis and the patient was started on oral antibiotics. Since the patient did not respond, lymph node biopsy was done and the histological features
\end{abstract}

Sudhakar $\mathrm{MK}^{1}$, Sathyamurthy $\mathrm{P}^{2}$, Indhumathi $\mathrm{E}^{3}$, Amarabalan Rajendran ${ }^{4}$, Bavya Vivek ${ }^{5}$

Affiliations: ${ }^{1}$ Professor, Department of Medicine, Sri Ramachandra University, Chennai, Tamilnadu, India;

${ }^{2}$ Assistant Professor, Department of Medicine, Sri Ramachandra University, Chennai, Tamilnadu, India;

${ }^{3}$ Associate Professor, Department of Medicine, Sri Ramachandra University, Chennai, Tamilnadu, India;

${ }^{4}$ Medical Officer, Department of Endocrinology Diabetes \& Metabolism, Sri Ramachandra University, Chennai, Tamilnadu, India; ${ }^{5}$ Post Graduate student, Department of Medicine, Sri Ramachandra University, Chennai, Tamilnadu, India.

Corresponding Author: Dr. Amarabalan Rajendran

No.99, Madam Street, Pillayar palayam,

Kanchipuram, Tamilnadu - 631501, INDIA;

Ph: +919940412624; Email: amarabalan@gmail.com

Received: 15 December 2010

Accepted: 23 January 2011

Published: 28 February 2011 suggested the diagnosis of Kikuchi's disease. Immunohistochemistry confirmed the diagnosis. The Patient was treated symptomatically and complete remission occurred in few weeks. Conclusion: Although the incidence of Kikuchi-Fujimoto disease is rare, clinicians should be aware of this condition as early recognition of the disease will minimize potentially harmful and unnecessary evaluations and treatments.

Keywords: Kikuchi-Fujimoto disease, Histiocytic Necrotizing Lymphadenitis, Systemic Lupus Erythematosus, Immunohistochemistry, Karyorrhexis

$* * * * * * * * *$

Sudhakar M K, Sathyamurthy P, Indhumathi E, Rajendran A, Vivek B. Kikuchi's disease: A case report from south India. International Journal of Case Reports and Images 2011;2(2):15-18.

$$
* * * * * * * * * \text {. }
$$

doi:10.5348/ijcri-2011-02-20-CR-4

\section{INTRODUCTION}

Kikuchi-Fujimoto disease (KFD) or histiocytic necrotizing lymphadenitis is an uncommon, idiopathic, generally self-limited cause of lymphadenitis $[1,2]$. Kikuchi first described the disease in 1972 in Japan. Fujimoto and colleagues independently described Kikuchi's disease in the same year. The cause of Kikuchi-Fujimoto disease is unknown. Some kind of viral or post viral etiology has been proposed. There have also been reports of a possible link between KFD and systemic lupus erythematosus (SLE). KikuchiFujimoto disease is an extremely rare disease. Its 
incidence has been reported worldwide with a higher prevalence among Japanese and other Asiatic individuals. KFD is more common in females compared to males with a male to female ratio of 1:4. People under 30 years of age are more affected by this disease than any other age group [3].

\section{CASE REPORT}

A 25-year-old female of south Indian origin presented to us with multiple neck swellings, fever, and polyarthritis of 15 days duration. There was no weight loss. There was no previous history of tuberculosis or contact with tuberculosis. She did not have history of any drug intake or atopy. She did not have any other significant medical problems.

Clinical Examination revealed bilateral large, mobile and tender-cervical lymphadenopathy larger node being the left supraclavicular lymph node which measured about $3 \times 5 \mathrm{~cm}$. Other enlarged nodes were left posterior cervical $(2 \times 2 \mathrm{~cm})$, left posterior auricular $(2 \times 2 \mathrm{~cm})$, left submandibular $(2 \times 2 \mathrm{~cm})$ and right submandibular $(1 \times 1 \mathrm{~cm})$. Lymph nodes were not palpable in other parts of the body. The blood pressure was $128 / 86 \mathrm{~mm} \mathrm{Hg}$ and the pulse rate was $98 / \mathrm{min}$. Her cardiovascular, respiratory and neurological examination was normal. The abdomen was soft with normal bowel sounds. Skin examination and Throat examination was also normal. Routine hematological parameters like hemoglobin, complete blood count, peripheral smear were within normal limits. ESR was $20 \mathrm{~mm} / 1^{\text {st }} \mathrm{hr}$. Blood glucose, urea, creatinine, sodium, potassium and bicarbonate levels were normal. Renal and liver function tests were normal. Blood and urine cultures were negative. Montoux showed induration of $4 \mathrm{~mm}$. Ultrasound abdomen and Chest radiograph was normal. Antinuclear antibody (ANA) and anti-DNA antibody were negative. Staining for AFB (acid-fast bacilli) was also negative.

Fine needle aspiration cytology (FNAC) of the left posterior cervical node showed features suggestive of reactive lymphadenitis and the patient was started on oral antibiotics. Since the patient continued to have fever and persistent lymphadenopathy, in spite of one week of antibiotics, lymph node biopsy was done and the histological features suggested the diagnosis of Kikuchi-Fujimoto disease. (Figure 1 and 2) Immunohistochemistry was also done. (Figure 3) The Patient was treated symptomatically with nonsteroidal anti-inflammatory drugs and the lymph nodes regressed in four weeks.

\section{DISCUSSION}

Kikuchi's disease most often presents with cervical lymphadenopathy which may be tender and can be accompanied by fever, upper respiratory tract

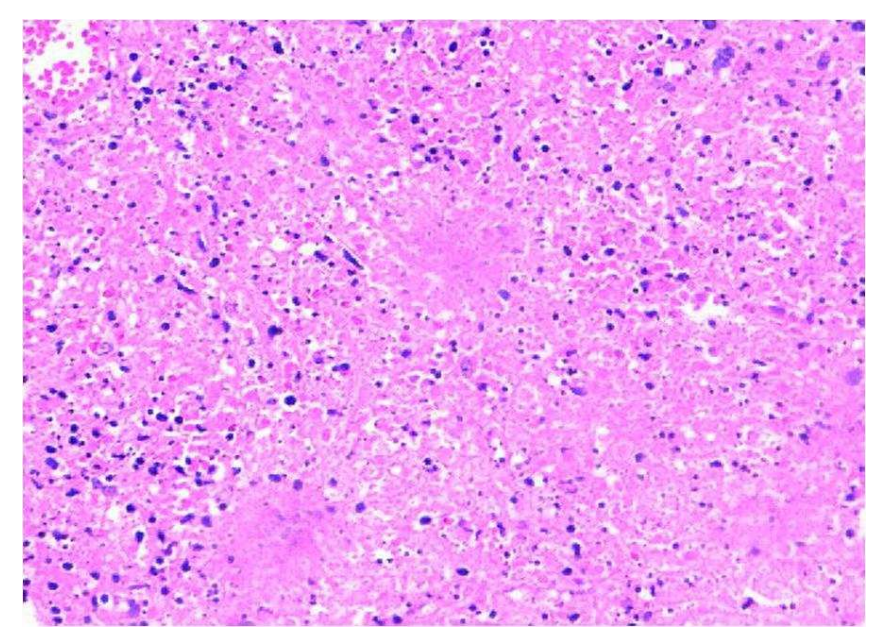

Figure 1: Lymph node biopsy section showing patchy areas of necrosis, proliferation of pale histiocytes, increase number of apoptotic cells, cellular debris and nuclear dust (karyorrhexis) (H\&E, x100)

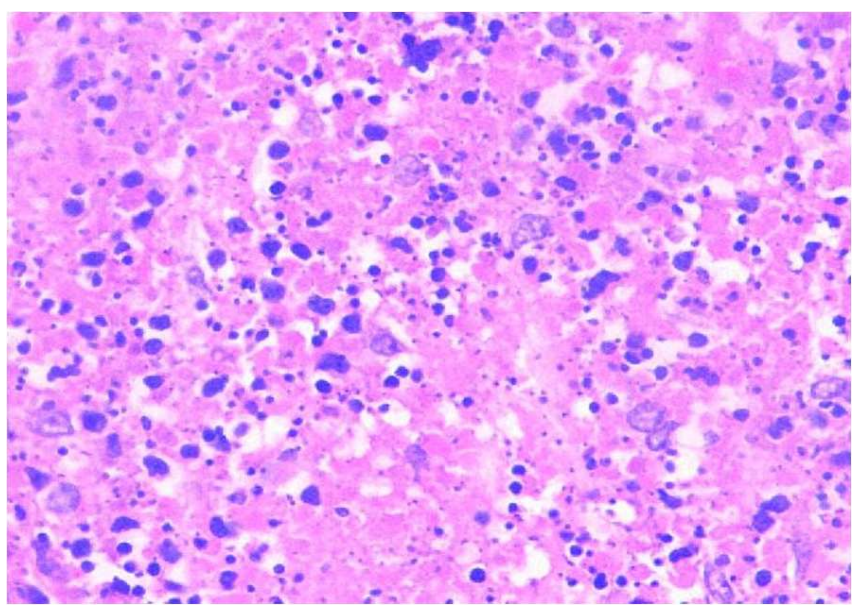

Figure 2: Lymph node biopsy section showing prominance of apoptotic cells, cellular debris and nuclear dust (karyorrhexis) (H\&E, x200)

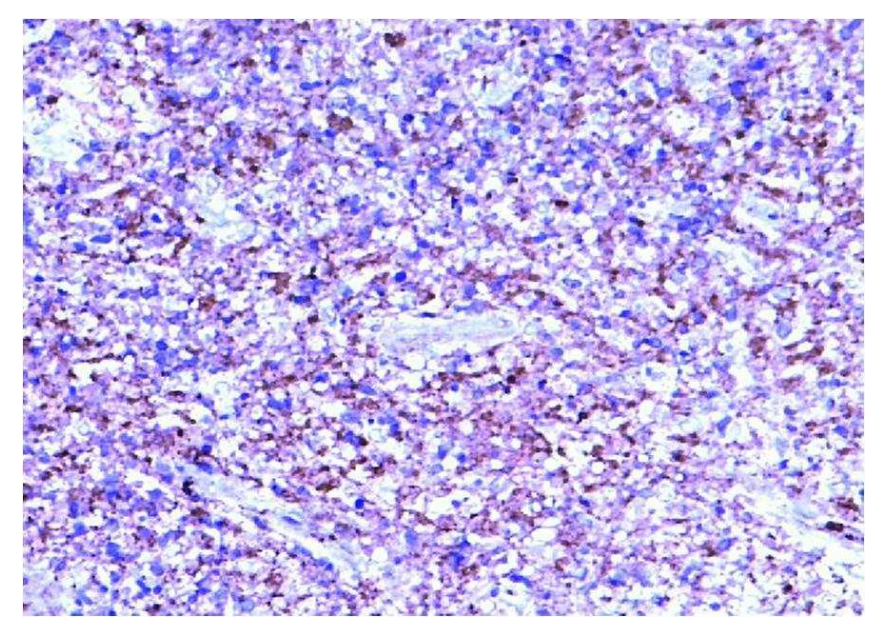

Figure 3: Immunohistochemistry showing CD68 positive histiocytes.. CD2O positive 'B' cells were also present in the preserved lymphoid areas. 
symptoms. Less common symptoms include arthralgia, skin rashes, weakness and night sweats. Weight loss, diarrhea, anorexia, chills, nausea, vomiting, chest and abdominal pain have also been reported. Some patients may also have hepatosplenomegaly. The exact etiology of Kikuchi's disease is not known. Viral agents such as Epstein barr virus (EBV), Human immunodeficiency virus (HIV), Herpes simplex virus, dengue virus, Human $\mathrm{T}$ lymphotrophic virus 1 (HTLV1) and Parvovirus B19 have been suggested as possible etiological agents, but none have been confirmed so far. Toxoplasma and other bacterial agents like Yersinia enterocolitica, Bartonella, Brucella have also been implemented [4]. An autoimmune mechanism has also been proposed because KFD is seen in conjunction with systemic lupus erythematosus (SLE). There are several reports suggesting an association between Kikuchi's disease and systemic lupus erythematosus (SLE). However no convincing evidence is available to confirm such association. The pathogenesis of Kikuchi's disease is still not fully understood. It is supposed that the primary event may be the activation of $\mathrm{T}$ lymphocytes and histiocytes. Proliferating $\mathrm{T}$ cells enter the cycle of apoptosis, which may form the areas of necrosis in lymph nodes and then the cellular debris is removed by histiocytes [5].

Routine laboratory investigations usually does not aid in the diagnosis except for erythrocyte sedimentation rate (ESR) and C-reactive protein (CRP) which might be elevated in some patients and many patients have a low white blood count. Moreover, 25\% to $31 \%$ of patients have atypical peripheral blood lymphocytes [6]. Fine-needle aspiration cytology (FNAC) only has a limited role in establishing the diagnosis of Kikuchi's disease with the overall diagnostic accuracy estimated at 56\% [4]. Diagnosis is based on histopathalogical findings of a lymph node biopsy. Morphologically, it is characterized by irregular paracortical areas of coagulative necrosis with abundant karyorrhectic debris, which can distort the nodal architecture, and large number of different types of histiocytes at the margin of the necrotic areas. The karyorrhectic foci are formed by different cellular types, predominantly histiocytes and plasmacytoid monocytes but also immunoblasts and small and large lymphocytes. Neutrophils are characteristically absent and plasma cells are either absent or scarce. The immunophenotype of Kikuchi's disease is primarily composed of mature CD8-positive and CD4-positive T lymphocytes. High rate of apoptosis is also seen among lymphocytes and histiocytes. The histiocytes express histiocyte-associated antigens such as lysozyme, myeloperoxidase (MPO) and CD68 which can be detected by immunohistochemistry. Plasmacytoid monocytes are also positive for CD68 but not for myeloperoxidase [7].

Clinically Kikuchi's disease may mimic systemic lupus erythematosus (SLE) or lymphoma (especially Tcell non- Hodgkins lymphoma) as both these diseases can present with lymphadenopathy and fever and the skin lesions of Kikuchi's disease patients can resemble those seen in SLE. Careful histopathologic examination will thus help us distinguish KFD from other diseases. Histological feature which helps in the differentiation of KFD from the lymphadenopathy of systemic lupus erythematosus is almost total absence of plasma cells in the involved nodal tissue. Moreover appropriate serologic tests should be done to exclude systemic lupus erythematosus [8]. Antinuclear antibodies (ANA) and anti-DNA antibodies were done in our patient and were negative. Features that distinguish KFD from malignant lymphoma include incomplete architectural effacement with patent sinuses, presence of numerous reactive histiocytes, relatively low mitotic rates, absence of Reed-Sternberg cells [7].

No specific treatment is available for Kikuchi's disease. Treatment is generally supportive. Nonsteroidal anti-inflammatory drugs (NSAIDs) may be used to alleviate lymph node tenderness and fever. The use of corticosteroids has been recommended in severe form of disease [9]. Intravenous Immunoglobulin has also been tried with some success [10]. The disease usually runs a benign course and the condition is self-limiting, usually resolves in several weeks to months. The disease has a recurrence rate of $3 \%$ to $4 \%[4]$.

\section{CONCLUSION}

Although the incidence of Kikuchi-Fujimoto disease is rare, this disorder must be considered among the differential diagnosis when a young female patient presents with fever and cervical lymphadenopathy. Clinically Kikuchi's disease may mimic lymphoma or systemic lupus erythematosus (SLE). Therefore a careful histopathological examination is necessary in arriving at the diagnosis. Early recognition of the disease is of crucial importance in minimizing potentially harmful and unnecessary evaluations and treatments.

$$
* * * * * * * * *
$$

\section{Author Contributions}

Sudhakar M K - Conception and design, Analysis and interpretation of data, Critical revision of the article, Final approval of the version to be published

Sathyamurthy P - Acquisition of data, Drafting the article, Final approval of the version to be published Indhumathi E - Acquisition of data, Drafting the article, Final approval of the version to be published Amarabalan Rajendran - Analysis and interpretation of data, Drafting the article, Critical revision of the article, Final approval of the version to be published Bavya Vivek - Acquisition of data, Drafting the article, Final approval of the version to be published

\section{Guarantor}

The corresponding author is the guarantor of 
submission.

\section{Conflict of Interest}

Authors declare no conflict of interest.

\section{Copyright}

(C) Amarabalan Rajendran et. al. 2010; This article is distributed under the terms of Creative Commons attribution 3.0 License which permits unrestricted use, distribution and reproduction in any means provided the original authors and original publisher are properly credited. (Please see www.ijcasereportsandimages.com /copyright-policy.php for more information.)

\section{REFERENCES}

1. Kikuchi M. Lymphadenitis showing focal reticulum cell hyperplasia with nuclear debris and phagocytes: a clinicopathological study. Acta Hematol Jpn 1972;35:379-380.

2. Fujimoto Y, Kozima Y, Yamaguchi K. Cervical subacute necrotizing lymphadenitis: a new clinicopathologic entity. Naika 1972;20:920-927.

3. Kuo T. Kikuchi's disease (histiocytic necrotising lymphadenitis): a clinicopathologic study of 79 cases with an analysis of histologic subtypes, immunohistology and DNA ploidy. Am J Surg Pathol 1995;19:798-809.

4. Sousa Ade A, Soares JM, de Sa Santos MH, Martins MP, Salles JM. Kikuchi-Fujimoto disease: three case reports. Sao Paulo Med J. 2010;128(4):232-235.

5. Hrycek A, Cieslik P, Witold S, Jacek P. KikuchiFujimoto disease: a case report. Rheumatol Int 2005 26:179-181.

6. Mosharraf-Hossain AK, Datta PG, Amin AS, Uddin M J. Kikuchi-Fujimoto Disease presenting with fever, lymphadenopathy and dysphagia. J Pak Med Assoc 2008;58:647-649.

7. Bosch X, Guilabert A. Kikuchi-Fujimoto disease. Orphanet J Rare Dis. 2006;1: 18. doi:10.1186/ 1750-1172-1-18.

8. Louis N, Hanley M, Davidson NM. KikuchiFujimoto disease: a report of two cases and an overview. The Journal of Laryngology Otology 1994;108:1001-1004. doi:10.1017/ So022215100128749.

9. Jang YJ, Park KH, Seok HJ. Management of Kikuchi's disease using glucocorticoid. J Laryngol Otol 2000;114:709-711.

10. Noursadeghi M, Aqel N, Gibson P, Pasvol G. Successful treatment of severe Kikuchi's disease with intravenous immunoglobulin Rheumatology 2005;45:235-237. 